Revue de droit comparé du travail et de la sécurité sociale

3 | 2021

Droits du travail et systèmes nationaux de protection sociale au prisme de la crise sanitaire. Adaptations ou changements profonds?

\title{
Les nouveaux joyaux de la protection sociale helvétique
}

\section{Anne-Sylvie Dupont}

\section{OpenEdition \\ Journals}

Édition électronique

URL : https://journals.openedition.org/rdctss/2220

DOI : $10.4000 /$ rdctss. 2220

ISSN : 2262-9815

Éditeur

Centre de droit comparé du travail et de la sécurité sociale

Édition imprimée

Date de publication : 1 novembre 2021

Pagination : 218-225

ISSN : 2117-4350

Référence électronique

Anne-Sylvie Dupont, "Les nouveaux joyaux de la protection sociale helvétique », Revue de droit comparé du travail et de la sécurité sociale [En ligne], 3 | 2021, mis en ligne le 01 novembre 2021, consulté le 19 novembre 2022. URL : http://journals.openedition.org/rdctss/2220 ; DOI : https:// doi.org/ $10.4000 /$ rdctss. 2220

\section{(c)}

Creative Commons - Attribution - Pas d'Utilisation Commerciale - Pas de Modification 4.0 International - CC BY-NC-ND 4.0

https://creativecommons.org/licenses/by-nc-nd/4.0/ 


\section{ANNE-SYLVIE DUPONT}

FACUltés de dRoIt de Neuchâtel ET GENÈVE

\section{LES NOUVEAUX JOYAUX DE LA PROTECTION SOCIALE HELVÉTIQUE*}

En Suisse, les années 2019 et 2020 ont été marquées par l'aboutissement de plusieurs projets législatifs importants, entrés en vigueur au début de cette année. Le plus médiatisé est sans conteste celui sur le congé de paternité (I).

Mais les nouvelles lois visant une meilleure conciliation de la vie familiale et professionnelle (II) et l'instauration d'une rente-pont pour les chômeuses et les chômeurs âgés (III) tiennent également le haut du pavé.

Il s'agit ici de présenter, dans les grandes lignes, ces nouvelles prestations sociales, tout en soulevant, ponctuellement, quelques éléments de réflexion.

\section{I - LE CONGÉ DE PATERNITÉ}

Le 27 septembre 2020, le peuple suisse a accepté, à un peu plus de 60\% des voix exprimées, de modifier la loi fédérale sur les allocations pour perte de revenu en cas de service et de maternité ${ }^{(L A P G)}{ }^{2}$, pour que les pères bénéficient désormais de deux semaines de congé a près la naissance d'un enfant. Cette modification est entrée en vigueur au $1^{\text {er janvier } 2021 .}$

Cette nouvelle prestation est garantie, comme le congé de maternité, par le truchement des allocations pour perte de revenu. Son objectif est avant toute chose de garantir l'effectivité du congé de paternité dans les relations de travail.

Ainsi, le nouvel art. $329 \mathrm{~g}$ al. 1 du Code des obligations $(\mathrm{CO})^{3}$ prévoit qu'en cas de paternité, le travailleur a droit à un congé de deux semaines. Il faut toutefois qu'il s'agisse soit du père légal de l'enfant au moment de sa naissance soit qu'il le devienne dans les six mois qui suivent cet évènement. Le congé doit être pris dans les six mois qui suivent la naissance de l'enfant (art. $329 \mathrm{~g}$ al. $2 \mathrm{CO}$ ).

* Ce texte se base sur une précédente publication, parue à la revue Plaidoyer 6/38 (2020), pp. 26-31, dont certains passages ont été repris.

1 Qui s'appellera désormais « loi fédérale sur les allocations pour perte de gain en cas de service, de maternité et de paternité ».

2 RS 834.1.

3 Loi fédérale du 30 mars 1911 complétant le Code civil suisse (Livre cinquième : Droit des obligations) (RS 220). 
Contrairement à la mère, le père n'est pas obligé de bénéficier de son congé d'un bloc, mais peut le prendre sous forme de semaines ou de journées isolées (art. $329 \mathrm{~g}$ al. 3 CO). Si le congé de paternité ne devient pas une période de protection durant laquelle le licenciement serait interdit ${ }^{4}$, il ne permet pas à l'employeur de diminuer le droit aux congés (art. $329 \mathrm{~b}$ al. 3 let. c CO).

Pendant la durée de ce congé, le père a droit à des prestations similaires à celles de la mère, soit des indemnités journalières couvrant $80 \%$ de son salaire, mais plafonnées à CHF 196.- par jour. Le droit à ces indemnités suppose, là encore, que le père soit légalement le père de l'enfant au moment de la naissance de ce dernier ou le devienne au cours des six mois qui suivent (art. 16i al. 1 let. a LAPG).

Outre les conditions générales d'affiliation au régime d'assurance (cf. art. 16i al. 1 let. b LAPG), le père doit, à la date de la naissance de l'enfant, exercer une activité lucrative, dépendante ou indépendante (art. $16 i$ al. 1 let. c LAPG).

Comme pour la mère, le décret d'application ${ }^{5}$ prévoit des règles ad hoc pour les pères qui ne remplissent pas ces conditions, soit en raison de la naissance prématurée de l'enfant (art. 16i al. 2 LAPG), soit en raison d'autres circonstances propres à la situation du père (art. 16i al. 3 LAPG). Les indemnités peuvent être perçues dans un délai-cadre de six mois qui commence à courir le jour de la naissance (art. 16j LAPG).

Contrairement aux mères, dont le droit aux indemnités journalières s'éteint si elles reprennent le travail, les pères peuvent bénéficier de leurs indemnités de paternité soit en une fois, soit de manière fractionnée. Les indemnités sont alors versées en fonction des jours de congé effectivement pris ; pour cinq indemnités perçues, deux indemnités supplémentaires sont versées ${ }^{6}$.

Le droit aux indemnités journalières prend fin si le père ou si l'enfant décède, ou encore si la filiation paternelle s'éteint par jugement (art. 16j al. 3 let. e LAPG). La mise en œuvre de cette dernière règle est de nature à poser quelques problèmes en pratique.

En effet, le droit suisse prévoit qu'une action en désaveu de paternité peut être intentée notamment par l'enfant, au plus tard dans l'année qui suit son accession à la majorité (art. $256 \mathrm{c}$ al. $2 \mathrm{CC})^{7}$.

Il est ainsi peu vraisemblable qu'un tel jugement intervienne dans les six mois qui suivent la naissance de l'enfant, de sorte qu'il est possible que le père désavoué ait perçu des indemnités de paternité qu'il lui appartiendra alors de rembourser.

Le financement de cette nouvelle prestation est assuré par une faible hausse des cotisations sociales prévues par ce régime d'assurance.

4 Alors que le congé de maternité représente une telle période pour la mère, cf. art. $336 c$ al. 1 let. c CO.

5 Règlement du 24 novembre 2004 sur les allocations pour perte de gain (RAPG ; RS 834.11). Cf. art. 29 et 30.

6 Ainsi, si le père prend quatre jours de congé, il touche quatre indemnités ; s'il prend cinq jours de congé, il en touche sept.

7 Code civil suisse du 10 décembre 1907 (RS 210). 


\section{II - LA CONCILIATION DE L'ACTIVITÉ PROFESSIONNELLE ET DE LA PRISE EN CHARGE DE PROCHES}

En Suisse, les proches aidants, dontl'engagement est pourtant reconnu et régulièrement salué par les autorités, ne bénéficient pas d'aménagements particuliers, ni sous l'angle de leurs conditions de travail, ni sous celui des prestations sociales.

Jusqu'au 31 décembre $2020^{8}$, ils ne pouvaient compter que sur un bonus prévu dans le premier pilier du système de pensions, permettant éventuellement une légère augmentation de la rente de vieillesse ${ }^{9}$ et, en fonction de leur canton de domicile, d'une compensation en principe partielle de leur perte de gain ${ }^{10}$.

Pour remédier à cette situation, le Parlement a adopté, le 20 décembre 2019, une loi intitulée « loi fédérale sur l'amélioration de la conciliation entre activité professionnelle et prise en charge de proches ${ }^{11}$. Cette loi a entraîné un certain nombre de modifications de la législation sociale, pour améliorer la situation des proches aidants. Ces modifications sont entrées en vigueur en deux temps.

\section{A - LES MESURES ENTRÉES EN VIGUEUR AU $1^{\text {ER }}$ JANVIER 2021}

\section{1 - L'élargissement des conditions d'octroi de la bonification pour tâches d'assistance}

Une première mesure est l'élargissement des situations donnant droit à la bonification pour tâches d'assistance. Depuis le 1 er janvier 2021, il suffit que la personne bénéficiant de l'aide d'un proche perçoive une allocation pour impotence de degré faible pour que l'aidant se voit créditer la bonification, dans la mesure où les autres conditions sont remplies. Jusqu'au 31 décembre 2020, l'allocation devait être allouée en raison d'une impotence de degré moyen au moins.

Par ailleurs, l'aide apportée au partenaire - à savoir la concubine ou le concubin ${ }^{12}$ permet désormais également de bénéficier de la bonification, pour autant que le ou la proche fasse ménage commun avec lui ou elle depuis au moins 5 ans sans interruption.

Pour mémoire, la bonification pour tâches d'assistance vient augmenter le revenu annuel moyen (RAM) qui sert ensuite à calculer le montant de la rente de vieillesse du premier pilier (AVS). Si la ou le proche a déjà atteint l'âge donnant droit à la rente de vieillesse au moment où l'aide est apportée, elle ou il ne tire aucun bénéfice de la bonification.

8 Pour un aperçu plus complet de la situation jusqu'à cette date, voir A.-S. Dupont, « Les aidants et le droit suisse de la sécurité sociale », in A. Cappellari (éd.), Les proches aidants saisis par le droit : regards franco-suisses, Aix-en-Provence 2018, p. 151.

9 II s'agit de bonifications pour tâches d'assistance (cf. art. 29septies LAVS de la loi fédérale du 20 décembre 1946 sur l'assurance-vieillesse et survivants [LAVS ; RS 831.10]).

10 Une telle prestation peut être prévue par les cantons dans le cadre du droit aux remboursement des frais de maladie prévu par la loi fédérale du 6 octobre 2006 sur les prestations complémentaires à I'AVS et à I'AI (LPC; RS 831.30).

11 FF 20198195 ss.

12 Voir le message du Conseil fédéral du 22 mai 2019 concernant la loi fédérale sur l'amélioration de la conciliation entre activité professionnelle et prise en charge de proches, FF 2019 3941, 3976 et $3996 \mathrm{~s}$. 


\section{2 - Le maintien du droit à l'allocation pour personne impotente en cas de séjour hospitalier prolongé}

Un enfant qui a besoin d'aide de façon permanente pour accomplir les actes essentiels de la vie quotidienne peut, comme un adulte, être mis au bénéfice d'une allocation pour impotent de l'assurance-invalidité s'il remplit les conditions de cette prestation ${ }^{13}$. En fonction de sa situation, il peut alors bénéficier, en sus, d'un supplément pour soins intenses (art. 42 $2^{\text {ter }}$ al. 3 LAl) ${ }^{14}$ et d'une contribution d'assistance (art. 42quater LAl et 39 a RAI) ${ }^{15}$.

Le droit à ces deux dernières prestations est lié au bénéfice de la première. Si l'allocation pour impotent est supprimée, même temporairement, le droit au supplément pour soins intenses et à la contribution d'assistance tombe également.

L'art. 67 al. 2 de la loi sur la partie générale du droit des assurances sociales ${ }^{16}$ prévoit la suppression du droit à l'allocation pour impotent pour chaque mois civil entier durant lequel le ou la bénéficiaire séjourne dans un établissement hospitalier aux frais de l'assurance sociale. Cette règle est de nature à pénaliser les familles dont un enfant est impotent, car le montant de leurs revenus peut fortement varier si ce dernier doit séjourner à l'hôpital.

En pratique, dans une telle situation, au moins l'un des deux parents diminue son taux d'activité pour assurer la prise en charge de l'enfant; en outre, il est fréquent que des tiers soient engagés contre rémunération pour relayer les parents. II s'agit d'engagements financiers qui, eux, ne disparaissent pas en cas de séjour de l'enfant à l'hôpital.

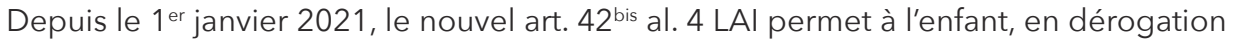
à l'article 67 al. 2 LPGA, de conserver son droit à l'allocation pour impotent - et donc aux autres prestations qui en découlent - s'il séjourne à l'hôpital plus d'un mois civil entier. Il sera néanmoins nécessaire d'attester que la présence régulière des parents ou de l'un des parents auprès de l'enfant hospitalisé est régulière et nécessaire.

En cas de séjour dans un home financé par l'enfant lui-même, respectivement par ses parents, le droit à l'allocation pour impotent est également maintenu, de même que le droit au supplément pour soins intenses (cf. art. $35^{\text {bis }}$ al. $2^{\text {ter }}$ et 36 al. 2 RAl).

\section{3 - Le calcul du droit aux prestations complémentaires (PC)}

Les PC sont des prestations versées aux personnes bénéficiant (notamment) d'une rente de vieillesse ou d'invalidité, dont les revenus sont insuffisants pour couvrir leur minimum vital. La différence entre leurs dépenses et leurs revenus (calculée strictement) est compensée par le versement d'une prestation financière.

13 Art. 42 s. LAl et 35 ss RAl. Sur l'évaluation de l'impotence chez l'enfant, cf. annexe III CIIAI.

14 Loi fédérale du 19 juin 1959 sur l'assurance-invalidité (RS 831.20).

15 Règlement du 17 janvier 1961 sur l'assurance-invalidité (RS 831.201).

16 Loi fédérale du 6 octobre 2000 sur la partie générale du droit des assurances sociales (LPGA ; RS 830.1). 
En principe, lorsqu'un ou une bénéficiaire de PC fait ménage commun avec d'autres personnes, le loyer est réparti à part égales entre tous les membres du ménage. La nouvelle loi a permis de modifier quelque peu cette répartition, de manière à éviter la pénalisation de la personne qui vit en communauté d'habitation avec une ou un proche aidant.

\section{4 - Le congé de courte durée}

Mentionnons encore que depuis le 1 er janvier 2021, le Code des obligations prévoit, dans les rapports de travail, un congé payé pour la prise en charge d'un membre de la famille ou du partenaire atteint dans sa santé. Le congé est limité au temps nécessaire à la prise en charge, mais ne doit pas dépasser trois jours par cas, et dix jours au total par année civile (art. $329 \mathrm{~g} \mathrm{CO}$ ).

Pour les travailleuses et les travailleurs employés par des entreprises soumises à la loi sur le travail $\left(\operatorname{LTr}^{17}\right)$, la présentation d'un certificat médical est obligatoire, et la limite absolue de dix jours par année civile ne vaut que dans la mesure où il ne s'agit pas de prendre en charge un enfant (art. 36 al. 3 et 4 LTr).

\section{B - LA MESURE ENTRÉE EN VIGUEUR AU $1^{\text {ER }}$ JUILLET 2021}

La mesure la plus importante prévue par la nouvelle loi est le congé de prise en charge d'un enfant gravement atteint dans sa santé. Cette mesure se décline d'abord en droit des assurances sociales : les nouveaux articles $16 i$ à $16 n$ LAPG prévoient un droit à des indemnités journalières couvrant $80 \%$ du salaire, plafonnées à CHF 196.- par jour (art. $16 \mathrm{~m}$ LAPG), pour les parents exerçant une activité lucrative qui interrompent cette activité pour prendre en charge l'enfant (art. 16i al. 1 LAPG).

La loi pose quatre conditions qui doivent être cumulativement remplies pour que l'enfant soit réputé gravement malade : il faut premièrement que son état de santé ait subi un changement majeur, que l'évolution ou l'issue de ce changement soit difficilement prévisible ou conduise à une atteinte durable ou croissante voire au décès de l'enfant, que l'enfant présente un besoin accru de prise en charge et que au moins l'un des deux parents doive interrompre son activité pour s'occuper de lui (art. 16j LAPG).

Le droit aux indemnités journalières est ouvert durant 98 jours - ou 14 semaines - sur une période globale de 18 mois courant depuis le jour où la première indemnité a été perçue (art. 16/ et 16k LAPG). Chaque cas ne donne droit qu'à une seule allocation (art. 16i al. 2 LAPG). Lorsque les deux parents exercent une activité lucrative, ils ont en principe droit chacun à la moitié des indemnités, mais peuvent convenir entre eux d'une autre répartition (art. 16/ al. 4 LAPG).

Lorsque le droit aux indemnités journalières selon les articles 16i à 16n LAPG est ouvert, l'employeur est obligé d'accorder à la mère ou au père un congé, dit congé de prise en charge, de 14 semaines au plus (art. $329 \mathrm{~h}$ al. $1 \mathrm{CO}$ ). Les modalités de ce congé sont calquées sur les conditions du droit aux indemnités journalières, notamment en ce qu'il peut être pris en une seule fois ou sous forme de journées isolées (art. 329h al. 4 CO).

17 RS 822.11. 
La période durant laquelle la travailleuse ou le travailleur bénéficie du congé de prise en charge est, passé le temps d'essai, une période de protection durant laquelle l'employeur ne peut pas résilier le contrat de travail. Cette protection est toutefois limitée à une période de six mois à compter du jour où la première indemnité journalière a été perçue (art. 336c al. 1 let. $c^{\text {bis }} \mathrm{CO}$ ).

\section{C - BRÈVE APPRÉCIATION DU NOUVEAU DISPOSITIF}

Lors de l'adoption de cette loi, des voix se sont fait entendre pour critiquer le fait que l'essentiel des mesures prises se limite aux personnes prenant en charge des enfants, sans davantage de considération pour les personnes accompagnant, notamment, des parents âgés. La critique est fondée, car leur rôle est primordial.

Néanmoins, il faut garder à l'esprit qu'en matière de protection sociale en Suisse, la politique des petits pas a toujours été la clé du succès, et que les projets trop ambitieux sont voués à l'échec. Dans ce sens, les nouveautés introduites, en particulier le congé de prise en charge, doivent être saluées.

\section{III - DES PRESTATIONS TRANSITOIRES POUR LES CHÔMEURS ÂGÉS}

À l'agenda politique de la Confédération et des cantons depuis plus de dix ans, la situation des personnes au chômage arrivant en fin de droit alors qu'elles sont proches de l'âge donnant droit à une rente de vieillesse $\mathrm{AVS}^{18}$ a été sensiblement améliorée grâce à

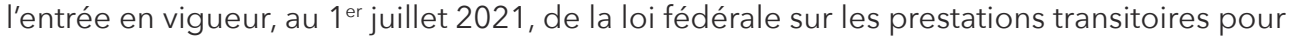
les chômeurs âgés (LPTRA) ${ }^{19}$. Cette loi prévoit des prestations destinées aux personnes qui arrivent en fin de droit à l'assurance-chômage ${ }^{20}$ après avoir atteint l'âge de 60 ans révolus (art. 3 al. 1 LPTRA) ${ }^{21}$.

En principe, ces prestations seront versées jusqu'à ce que le ou la bénéficiaire atteigne l'âge de la retraite selon l'AVS. Les prestations transitoires ayant uniquement pour objectif de couvrir les besoins vitaux, il sera demandé aux personnes qui, de toute manière, bénéficieront de prestations complémentaires (PC) une fois atteint l'âge de la retraite d'anticiper le versement de leur rente AVS 22 , et cesseront de toucher les prestations transitoires du moment que cette dernière leur sera versée (art. 3 al. 1 let. a et b LPTRA).

Le droit aux prestations transitoires est subordonné à la condition d'une affiliation à l'AVS durant 20 ans au moins, dont au moins cinq années après l'âge de 50 ans. Les personnes qui sollicitent ces prestations doivent soit avoir exercé une activité lucrative leur

18 Actuellement, 65 ans pour les hommes et 64 ans pour les femmes.

19 RS 8372.

20 Selon l'art. 3 al. 2 LPTRA, « une personne est arrivée en fin de droit lorsqu'elle a épuisé son droit aux indemnités de l'assurance-chômage ou lorsque son droit aux indemnités de l'assurance-chômage s'est éteint à l'expiration du délai-cadre d'indemnisation et qu'elle n'a pas pu ouvrir un nouveau délai-cadre d'indemnisation ».

21 L'art. 5 al. 1 let. a précise qu'il faut être arrivé en fin de droit au plus tôt pendant le mois au cours duquel l'on a atteint 60 ans.

22 Art. 40 LAVS et 56 s. RAVS (RS 831.101). 
procurant un revenu annuel couvrant $75 \%$ de la rente AVS maximale, soit CHF 2'390.- dès le 1 er janvier 2021 (art. 5 al. 1 let. b LPTRA).

Finalement, la fortune nette de la personne assurée ne doit pas dépasser la moitié des

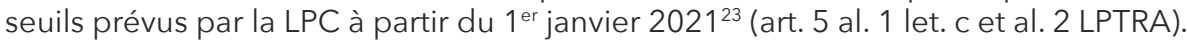

Calquées sur les prestations complémentaires à l'AVS/AI, les prestations transitoires se composent de la prestation annuelle (prestation en espèces) et du remboursement des frais de maladie et d'invalidité (prestation en nature). Elles sont subsidiaires aux autres prestations d'assurances sociales, dont les PC à l'AVS/AI (art. 6 et art. 5 al. 3 LPTRA). La prestation annuelle correspond à la part des dépenses reconnues qui excède les revenus déterminants (art. 7 al.1 LPTRA).

Le total des prestations versées (en nature et en espèces) sera cependant plafonné à un montant correspondant à un multiple du montant reconnu pour la couverture des besoins vitaux, soit à 2,25 fois CHF 19'490.- pour une personne seule ${ }^{24}$. Pour le reste, la mise en œuvre des nouvelles prestations transitoires est largement identique à celle des prestations complémentaires dans le régime du premier pilier.

Conformément à l'art. 5 al. 1 LPTRA, le droit aux prestations est réservé aux personnes qui ont leur domicile et leur résidence habituelle en Suisse. Dans la mesure où les nouvelles prestations transitoires doivent être qualifiées de prestations de préretraite au sens de l'art. 3 al. 1 let. i R (CE) n 883/200425, cette clause de résidence n'est pas opposable aux ressortissants suisses ou UE/AELE qui sont domiciliés sur le territoire d'un pays membre de I'UE/AELE26, notamment aux travailleuses et travailleurs frontaliers. Il est donc prévu dans la nouvelle loi que si les prestations transitoires doivent être versées dans l'un de ces Etats, leurs bases de calcul (dépenses reconnues et revenus déterminants) soient adaptées en fonction du pouvoir d'achat de l'Etat de résidence (art. 8 LPTRA).

\section{Conclusion}

Le congé paternité, les prestations pour les proches aidants et les prestations transitoires pour les chômeurs âgés représentent assurément des avancées sociales importantes, et ce serait faire "la fine bouche » que de regretter leur entrée en vigueur. A l'inverse en tirer des conclusions trop réjouissantes pour le débat social en Suisse serait tout aussi dangereux.

En premier lieu, il faut en effet souligner que le congé paternité et les prestations pour les proches aidants ne sont pas à proprement parler des mécanismes solidaires. L'un comme les autres sont principalement financés par une solution de mutualisation qui ne consent pas d'avantage particulier en faveur des personnes économiquement défavorisées. En revanche, les prestations transitoires pour les chômeurs âgés seront financées par les

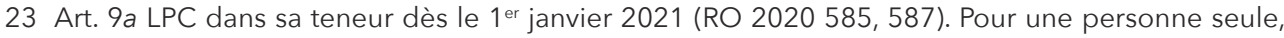
la limite de fortune pour avoir droit aux prestations transitoires sera ainsi de CHF 50'000.-. Le bien immobilier servant d'habitation à son propriétaire ne sera pas pris en compte dans la fortune nette.

24 Art. 7 al. 2 cum art. 9 al. 1 let. a LPTRA.

25 Message du Conseil fédéral du 30 octobre 2019 concernant la loi fédérale sur les prestations transitoires pour les chômeurs âgés, FF 2019 7797, 7856 s.

26 Art. 7 du Règlement (CE) n 883/2004 du Parlement européen et du Conseil du 29 avril 2004 portant sur la coordination des systèmes de sécurité sociale. 
deniers publics. Il s'agit donc véritablement d'une démarche solidaire, en ce sens qu'elles seront majoritairement financées par les personnes qui risquent d'en profiter le moins

En second lieu, il faut signaler que le $1^{\text {er }}$ janvier 2021 est également entrée en vigueur une réforme de la loi sur les prestations complémentaires dans le régime du premier pilier, qui durcit les conditions d'accès à ces prestations qui représentent, pour les personnes assurées concernées, le dernier rempart contre la pauvreté.

La large acceptation du congé de paternité et l'absence totale de débat politique au sujet de la loi sur l'amélioration de la conciliation entre activité professionnelle et prise en charge de proches, lues en parallèle avec la révision de la loi sur les prestations complémentaires, posent la question d'une possible gentrification de la protection sociale helvétique. 